\title{
Nanoscale pressure effects in individual double-wall carbon nanotubes
}

\author{
P. Puech,,${ }^{1} *$ E. Flahaut ${ }^{2}$ A. Sapelkin, ${ }^{3}$ H. Hubel, ${ }^{3}$ D. J. Dunstan, ${ }^{3}$ G. Landa,${ }^{4}$ and W. S. Bacsa ${ }^{1}$ \\ ${ }^{1}$ LPST, UMR-CNRS 5477, IRSAMC, Université Paul Sabatier, 118 Route de Narbonne, 31062 Toulouse, France \\ ${ }^{2}$ CIRIMAT-LCMIE, UMR CNRS 5085, Université Paul Sabatier, 118 Route de Narbonne, 31077 Toulouse Cedex 4, France \\ ${ }^{3}$ Physics Department, Queen Mary, University of London, Mile End Road, London E1 4NS, United Kingdom \\ ${ }^{4}$ LAAS-CNRS UPR8001, 7 Avenue du Colonel Roche, 31077 Toulouse Cedex 4, France
}

(Received 22 May 2006; published 30 June 2006)

\begin{abstract}
We use the signal from the internal tubes of double-wall carbon nanotubes as an ideal pressure reference. The intensity associated with the $G$ band of the external tubes is shown to be related to the interaction of the pressure medium and the carbon nanotube. We observe clear pressure medium dependent pressure coefficients of the Raman $G$ band using atomic argon, oxygen, and alcohol. The $G$ band of the internal tubes shifts between 5.1 and $3.3 \mathrm{~cm}^{-1} / \mathrm{GPa}$ and for the external tubes between 5.8 and $8.6 \mathrm{~cm}^{-1} / \mathrm{GPa}$ for the different pressure media used. We find that the spectral shape of the optical phonon band depends clearly on the pressure medium. Ab initio calculations support local partial ordering and shell formation of the pressure medium around the nanotube. The shell formation around the tube has a strong impact on the local pressure transmission.
\end{abstract}

DOI: 10.1103/PhysRevB.73.233408

PACS number(s): $61.46 . \mathrm{Fg}, 63.22 .+\mathrm{m}, 61.50 . \mathrm{Ks}, 78.30 . \mathrm{Na}$

The small diameter, exceptional mechanical properties, and large surface area make carbon nanotubes (CNTs) particularly attractive for applications. ${ }^{1,2}$ Double-wall carbon nanotubes (DWCNTs), which is the simplest form of multiwall carbon nanotubes, ${ }^{3,4}$ give the opportunity to determine the influence of the environment of the external wall while using the internal wall as a reference. The internal wall experiences a smaller stress due to shielding by the external wall. ${ }^{5,6}$ Effects due to the pressure transmitting medium, in hydrostatic experiments, have been reported earlier and evaluation of hydrostatic pressure condition remains controversial. The broadening of the observed luminescence line (R1) of ruby crystals in the pressure medium is usually used to evaluate the deviation from hydrostatic pressure condition. Burnett et $\mathrm{al}^{7}$ observe no broadening of the R1 ruby line although the photoluminescence peak of a quantum well does broaden. Helium as a pressure transmitting medium gives no observable deviations from hydrostatic pressure conditions. We expect that the pressure transmission is different for nanoscale systems due to the finite molecular size of the medium or possible organization around the CNTs. We compare three different pressure media to test nanoscale pressure effects on the CNTs. We use argon, oxygen, and methanol-ethanol in the proportion 4:1. Recent studies of Merlen et al. ${ }^{8,9}$ on CNT bundles (CNTs grown by the arc discharge method) show no frequency dependence on the media. In this study we use CNTs grown using the catalytic chemical vapor deposition method (CCVD), which leads to the formation of individual CNT and small bundles allowing nanoscale pressure effects to be investigated. With the three different media we can test the interaction of hydrogen end groups, physisorbed oxygen, and argon on CNTs. The interaction of $\mathrm{O}_{2}$ and Ar with CNTs has been studied in detail. ${ }^{10,11}$ The methanol-ethanol medium is used in several high pressure studies on CNTs. ${ }^{612-17}$ Argon gas adsorption studies on $\mathrm{CNTs}^{18}$ identified several adsorption sites: interstitial channels, external groove sites, and the external surface. Pseudohexagonal medium range ordering of adsorbents have been predicted on the external surface of CNTs. ${ }^{19}$ Oxygen physisorption on bundles has been considered by Ulbricht et al. ${ }^{10}$ who demonstrated that the physisorption is induced by van der Waals (vdW) interaction. Molecular oxygen solidifies with pressure and leads to two phase transitions at 6 and $10 \mathrm{GPa}$ at room temperature, ${ }^{20}$ which gives us the opportunity to observe the effect of solidification of the pressure medium on CNTs. We compare our experimental results with $a b$ initio calculations to get a better understanding of the influence of the pressure media on the pressure condition around the CNTs.

The DWCNTs were prepared by CCVD. ${ }^{21,22}$ Highresolution electron microscopy images show the presence of individual and small bundles of DWCNTs with radius ranging from 0.3 to $1.5 \mathrm{~nm}$. The CCVD method is associated to the individual growth of CNTs from catalytic particles formed in situ from the selective reduction of cobalt oxide to cobalt nanoparticules. The tubes agglomerate after the chemical removal of the catalyst through $\mathrm{vdW}$ interaction and form bundles. Frequent formation of interstitial channels are expected due to large diameter distribution. The tubes are single $(15 \%)$, double $(80 \%)$, or triple walled $(5 \%)$. A typical picture is reported in Fig. 1. Contrast analysis suggests the frequent formation of interstitial channels. Raman spectra were recorded at room temperature using a Renishaw Raman microprobe instrument. A $\times 20$ microscope objective was used to focus the laser beam $(633 \mathrm{~nm})$ on the sample inside the pressure cell. The laser output power was kept at $25 \mathrm{~mW}$. Heating effects are negligible at this wavelength and power level. ${ }^{23}$ The high-pressure Raman measurements were performed in a diamond anvil cell. The pressure was monitored using the luminescence of a ruby chip inside the cell. All $a b$ initio calculations have been carried out using VASP. ${ }^{24-27}$ The cell was composed of four carbon atoms with two neon atoms. The distance between the graphene sheet and the medium was kept constant. The equilibrium position was then determined by systematically exploring different configurations.

We present in Fig. 2 the Raman $G$ band of DWCNTs as a 


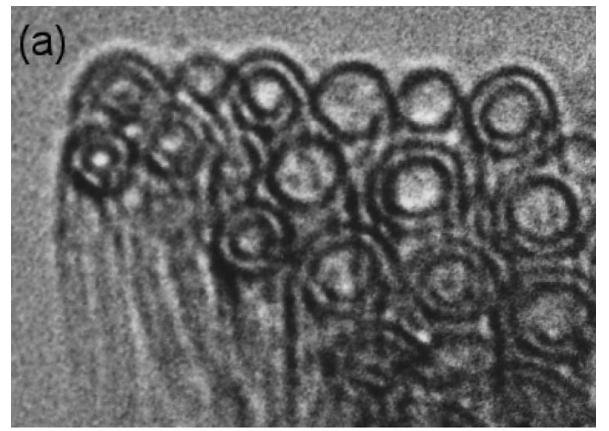

(b)

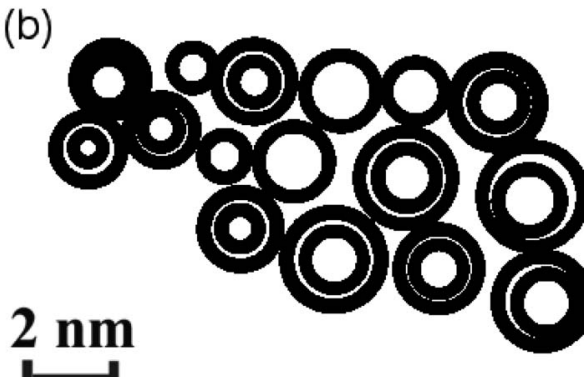

FIG. 1. TEM image [(a) — top] and contrast analysis [(b) bottom] showing a bundle composed of heterogeneous CNTs with large interstitial channels.

function of pressure using oxygen as pressure medium and we observed solidification at $6 \mathrm{GPa}$ by color change. No solidification was observed for methanol-ethanol and argon as pressure transmitting medium. In Fig. 2 above $6 \mathrm{GPa}$ the high energy $G$ band reduces its intensity and broadens into a single band. We fitted the spectra with three Lorentzians, one for the high energetic mode (HEM) located around $1550 \mathrm{~cm}^{-1}$, one for the $G$ band associated with the internal tubes around $1582 \mathrm{~cm}^{-1}$, and one for the $G$ band associated with the external tube around $1590 \mathrm{~cm}^{-1}$ at zero pressure. The fit is stable for the two $G$ bands but is less stable for the HEM band due to its low intensity. The shown fit has been obtained after full minimization independent of the starting parameters. The fact that the intensity and shape of the $G$ band spectrum does not change strongly indicates that resonance effects from the different diameter tubes are not dominant. We noticed that the ratio of the intensity of the $G$ band associated with the internal and external tubes is constant for each of the three pressure media when changing the pressure and listed the values in Table I. For oxygen, the pressure coefficient for the $G$ bands is constant up to $8 \mathrm{GPa}$ without any change when the oxygen solidification occurs. The
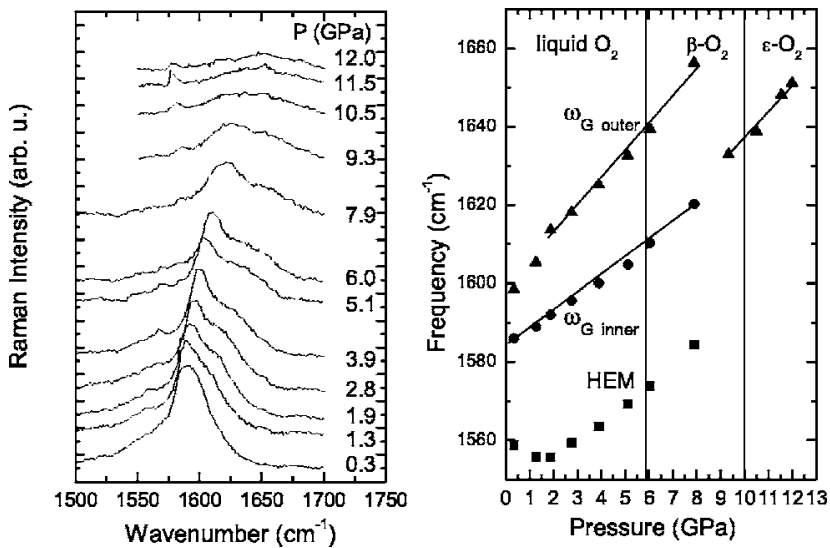

FIG. 2. Raman spectra (left) with $\mathrm{O}_{2}$ medium and frequency position (right) obtained by fitting with three Lorentzians in function of applied pressure.

pressure coefficient of the $G$ band of the external tubes is clearly larger than for the internal tubes. At high pressure $(>9 \mathrm{GPa}$ ) only one $G$ band is observed. No local freezing of the medium can be involved as no change after the solidification of the oxygen is observed.

Figure 3 shows the $G$ band of DWCNTs at $5 \mathrm{GPa}$ for the three pressure media and the three components of the fitted spectra. The pressure coefficients of the two $G$ bands are also indicated. Table I gives all the $G$ band positions and pressure coefficients for the three media. The ratio of the pressure coefficient for the internal tubes and the external tubes is the same (0.59) for all three pressure media. Interestingly, filled DWCNTs have a larger ratio of the pressure coefficients of the internal and external tubes. ${ }^{28}$ We assume a near to zero pressure inside the DWCNTs. Even in the case where a substantial fraction of the pressure medium penetrates into the tube due to open tube ends or defects in both walls, the small number of atoms involved leads to a negligible pressure inside DWCNTs. We note that the relative intensity of the three components changes consistently with the pressure medium and it appears that an intensity transfer from the $G$ mode of the external tubes to the HEM takes place. The HEM is smallest for methanol-ethanol and largest for argon as pressure transmitting medium. The HEM band has been observed in metallic CNTs and attributed to coupling of the optical phonons to a continuum of electronic states. The more intense and broader HEM for Ar indicates that the coupling of Ar with the CNT is stronger and the pressure medium increases the electron-phonon coupling.

In the sample used here, the large size distribution implies

TABLE I. Raman $G$ band positions, pressure coefficients, integrated intensity ratio at low pressure, and high pressure results of DWCNTs in $\mathrm{O}_{2}$, methanol-ethanol, and Ar.

\begin{tabular}{|c|c|c|c|c|c|c|c|c|}
\hline Medium & $\begin{array}{l}\omega_{\text {inner }}(P=0) \\
\left(\mathrm{cm}^{-1}\right)\end{array}$ & $\begin{array}{l}\omega_{\text {outer }}(P=0) \\
\left(\mathrm{cm}^{-1}\right)\end{array}$ & $\frac{d \omega_{\text {inner }}}{d p}$ & 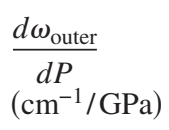 & $\frac{d \omega_{\text {inner }}}{d \omega_{\text {outer }}}$ & $\frac{L_{\text {inner }}}{I_{\text {outer }}}$ & $\begin{array}{l}P_{\text {transition }} \\
(\mathrm{GPa})\end{array}$ & $\frac{d \omega_{\text {after transition }}}{d P}$ \\
\hline Methanol-ethanol & 1582 & 1594 & 3.3 & 5.8 & 0.57 & 0.96 & 12 & 6 \\
\hline $\mathrm{O}_{2}$ & 1584 & 1598 & 4.1 & 6.9 & 0.59 & 1.56 & 9 & 7 \\
\hline Argon & 1581 & 1592 & 5.1 & 8.6 & 0.59 & 1.85 & 6 & 8.5 \\
\hline
\end{tabular}




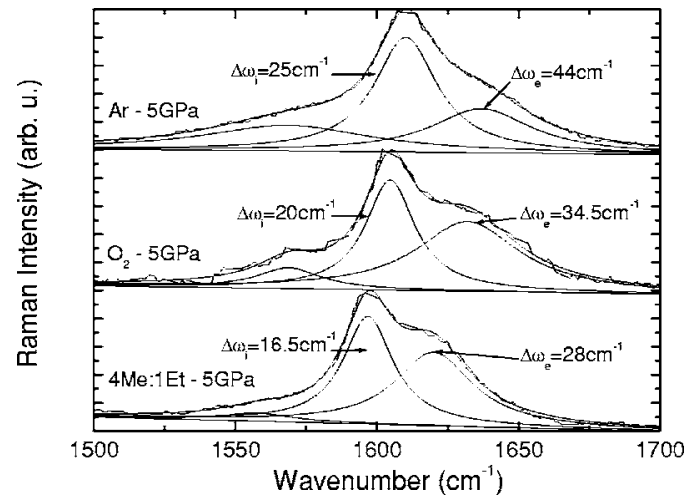

FIG. 3. Effect of the pressure medium on the CNT spectra. The frequency shift is given for both the $G$ band corresponding to the internal $(i)$ and external $(e)$ tubes.

a large number of helicities for both internal and external tubes. Consequently, the surfaces in contact between tubes are in register leading to weak adhesion. We expect that the medium surrounds the tubes. This is supported by the fact that in the case of tubes in a bundle (i.e., without surrounding pressure medium) no medium effect is observed. ${ }^{8}$ The medium dependence of the shape of the optical phonon band also indicated that the DWCNTs are surrounded by the pressure transmitting medium. We conclude that the DWCNTs here are surrounded by the pressure medium. The weak van der Waals interaction between the two walls has the effect that the radial stress components are continuous while the tangential stress component is discontinuous for the two walls. ${ }^{5}$ The different broadening of the two $G$ bands can be explained by the diameter size distribution. ${ }^{5}$ The mean internal radius is $0.7 \mathrm{~nm}$ and the mean external radius is $1.04 \mathrm{~nm}$ for the sample used here. Using the elastic constants of graphite $^{12,29}$ and considering the elastic continuum model for DWCNTs we find a pressure coefficient for the internal (external) tube of $-0.8 p(-1.7 p)$, which corresponds to $3.8 \mathrm{~cm}^{-1} / \mathrm{GPa}\left(8.1 \mathrm{~cm}^{-1} / \mathrm{GPa}\right)$. The pressure coefficients we observe for argon are close to these values and are within the same order of magnitude for the pressure media considered here. Ye et al. ${ }^{30}$ have used the Tersoff potential and molecular dynamics simulations to study the flattening of armchair DWCNTs. The flattening was found to depend strongly on the DWCNT diameter. For the DWCNT $(7,7) @(12,12)$ close to the mean diameter of the sample used here, we can estimate the pressure coefficient from the change of the calculated bond length: a splitting of the $G$ band is observed with a slope of 2.5 and $9.6 \mathrm{~cm}^{-1} / \mathrm{GPa}$. A flattening occurs at 3.5 GPa. The finite size of the molecules of the pressure medium has not been explicitly taken into account in the study. ${ }^{30}$ The pressure transition observed here and reported in Table I is clearly different. We find that for all three pressure media at about a $G$-band shift of $60 \mathrm{~cm}^{-1}$, the $G$-band splitting disappears. The same value is found for SWNTs. ${ }^{14} \mathrm{~A}$ $G$-band shift of $60 \mathrm{~cm}^{-1}$ corresponds for graphite to a pressure of $14 \mathrm{GPa}$. The phase transformation from graphite to diamond begins at this pressure..$^{31,32}$

To investigate more closely the differences observed with the three pressure media, we have carried out ab initio cal-

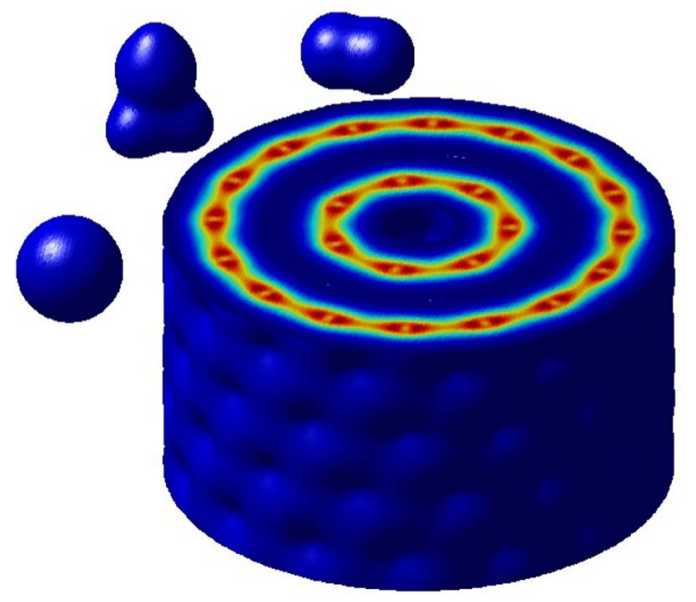

FIG. 4. (Color online) Electronic density at a van der Waals radius for Ar, methanol, $\mathrm{O}_{2}$, and DWCNT. The CNT surface is not uniform.

culations. Figure 4 plots the electronic density at a distance of the average van der Waals radius for the three molecules of the pressure medium and DWCNT. The DWCNT surface shows clearly a corrugation caused by the honeycomb lattice. We investigated the effect of the relative position of the atom or molecule of the pressure medium on the honeycomb lattice. We used the same density and applied the pressure (1) in the center of the carbon hexagon and (2) on the top of carbon atoms. We carried out ab initio calculations using $\mathrm{Ne}$ to study its interaction with a graphite sheet. Ne is similar to Ar apart from having a different van der Waals radius. The size of $\mathrm{Ne}$ is compatible with the honeycomb lattice of the CNT and we explore here only geometrical effects. We found that a decrease of $12 \%$ of the distance between a monolayer of $\mathrm{Ne}$ with a monolayer of graphene leads in all the cases to an in-plane deformation of less than $0.1 \%$. X-ray diffraction studies of graphite at $10 \mathrm{GPa}$ show that the lattice in-plane deformation is $1 \%$ with a lattice out of plane deformation of $12 \% .{ }^{29}$ This indicates that the lateral stress due to anisotropic interaction of the molecules of the pressure medium with the tube wall does not play an important role here. The density of the medium around the tube has already been investigated: Bienfait et $a l^{33}$ conclude from molecular dynamics simulations that argon adsorbs epitaxially on the hexagonal lattice of the tube walls. The density of Ar atoms around a tube is high. The uniform and epitaxial adsorption is expected to be more difficult to obtain with oxygen or alcohol molecules. Consequently, the pressure is transmitted less effectively in the case of oxygen and alcohol due to the structure formed around the tube by the first few layers. Evidence of $\mathrm{H}_{2} \mathrm{SO}_{4}$ partly ordered around CNTs has been recently reported ${ }^{34}$ and the formation of structured water and other species has been considered. ${ }^{33}$

In this paper, we present for the first time clear experimental results which show that surface interaction effects at the nanometric scale can lead to a change of up to $50 \%$ in the stress transmission using DWCNTs. We observe differences in the pressure behavior of DWCNTs as a function of pressure transmitting medium. Differences are explained by local 
ordering and shell formation of the pressure transmitting medium around the CNT. This result shows the importance of molecular organization at the surface of CNTs. Molecular adaptation to the surface is frequently encountered in biology and we show here that it is also observed for the pressure medium near CNTs. This has important consequences on the formation of ordered molecular phases around CNTs and the understanding of the interaction of CNTs in composites.
*Electronic address: pascal.puech@1pst.ups-tlse.fr

${ }^{1}$ M. S. Dresselhaus, G. Dresselhaus, and P. Avouris, Carbon Nanotubes: Synthesis, Structure, Properties and Applications, 1st ed. (Springer, Berlin, 2001).

${ }^{2}$ P. J. F. Harris, Carbon Nanotubes and Related Structures, 1st ed. (Cambridge University Press, Cambridge, 2002).

${ }^{3}$ S. Bandow, G. Chen, G. U. Sumanasekera, R. Gupta, M. Yudasaka, S. Iijima, and P. C. Eklund, Phys. Rev. B 66, 075416 (2002).

${ }^{4}$ S. Bandow, M. Takizawa, K. Hirahara, M. Yudasaka, and S. Iijima, Chem. Phys. Lett. 337, 48 (2001).

${ }^{5}$ P. Puech, H. Hubel, D. Dunstan, R. R. Bacsa, C. Laurent, and W. S. Bacsa, Phys. Rev. Lett. 93, 095506 (2004).

${ }^{6}$ J. Arvanitidis, D. Christofilos, K. Papagelis, K. S. Andrikopoulos, T. Takenobu, Y. Iwasa, H. Kataura, S. Ves, and G. A. Kourouklis, Phys. Rev. B 71, 125404 (2005).

${ }^{7}$ J. H. Burnet, H. M. Cheong, and W. Paul, Rev. Sci. Instrum. 61, 3904 (1990).

${ }^{8}$ A. Merlen, N. Bendiab, P. Toulemonde, A. Aouizerat, A. San Miguel, J. L. Sauvajol, G. Montagnac, H. Cardon, and P. Petit, Phys. Rev. B 72, 035409 (2005).

${ }^{9}$ A. Merlen, P. Toulemonde, N. Bendiab, A. Aouizerat, J. L. Sauvajol, G. Montagnac, H. Cardon, P. Petit, and A. San Miguel, Phys. Status Solidi B 243, 690 (2006).

${ }^{10}$ H. Ulbricht, G. Moos, and T. Hertel, Phys. Rev. B 66, 075404 (2002).

${ }^{11}$ S. Rols, M. R. Johnson, P. Zeppenfeld, M. Bienfait, O. E. Vilches, and J. Schneble, Phys. Rev. B 71, 155411 (2005).

${ }^{12}$ J. Sandler, M. S. P. Shaffer, A. H. Windle, M. A. Montes-Moran, C. A. Cooper, R. J. Young, and M. P. Halsall, Phys. Rev. B 67, 035417 (2003).

${ }^{13}$ U. D. Venkateswaren, A. M. Rao, E. Richter, M. Menon, A. Rinzler, R. E. Smalley, and P. C. Eklund, Phys. Rev. B 59, 10928 (1999).

${ }^{14}$ A. K. Sood, P. V. Teresdesai, D. V. S. Muthu, R. Sen, A. Govindaraj, and C. N. R. Rao, Phys. Status Solidi B 215, 393 (1999).

${ }^{15}$ M. J. Peters, L. E. McNeil, J. P. Lu, and D. Kahn, Phys. Rev. B
61, 5939 (2000)

${ }^{16}$ C. Thomsen, S. Reich, H. Jantoljak, I. Loa, K. Syassen, M. Burghard, G. S. Duesberg, and S. Roth, Appl. Phys. A: Mater. Sci. Process. 69, 309 (1999).

${ }^{17}$ J. R. Wood, M. D. Frogley, E. R. Meurs, A. D. Prins, D. J. Dunstan, and H. D. Wagner, High Press. Res. 18, 153 (2000).

${ }^{18}$ W. Shi and J. K. Johnson, Phys. Rev. Lett. 91, 015504 (2003).

${ }^{19}$ M. Bienfait, P. Zeppenfeld, N. Dupont-Pavlovsky, J. P. Palmari, M. R. Johnson, T. Wilson, M. DePies, and O. E. Vilches, Phys. Rev. Lett. 91, 035503 (2003).

${ }^{20}$ H. J. Jodl, F. Bolduan, and H. D. Hochheimer, Phys. Rev. B 31, 7376 (1985).

${ }^{21}$ R. R. Bacsa, Ch. Laurent, A. Peigney, W. S. Bacsa, Th. Vaugien, and A. Rousset, Chem. Phys. Lett. 323, 566 (2000).

${ }^{22}$ E. Flahaut, R. Bacsa, A. Peigney, and Ch. Laurent, Chem. Commun. (Cambridge) 12, 1442 (2003).

${ }^{23}$ A. Bassil, P. Puech, L. Tubery, W. Bacsa, and E. Flahaut, Appl. Phys. Lett. (to be published).

${ }^{24}$ G. Kresse and J. Hafner, Phys. Rev. B 47, 558 (1993).

${ }^{25}$ G. Kresse and J. Hafner, Phys. Rev. B 49, 14251 (1994).

${ }^{26}$ G. Kresse and J. Furthmuller, Comput. Mater. Sci. 6, 15 (1996).

${ }^{27}$ G. Kresse and J. Furthmuller, Phys. Rev. B 54, 11169 (1996).

${ }^{28} \mathrm{~J}$. Gonzalez et al. (private communication).

${ }^{29}$ M. Hanfland, H. Beister, and K. Syassen, Phys. Rev. B 39, 12598 (1989).

${ }^{30}$ X. Ye, D. Y. Sun, and X. G. Gong, Phys. Rev. B 72, 035454 (2005).

${ }^{31}$ T. Yagi, W. Utsumi, M. A. Yamakata, T. K. Kegawa, and O. Shimomura, Phys. Rev. B 46, 6031 (1992).

${ }^{32}$ R. Ahuja, S. Auluck, J. Trygg, J. M. Wills, O. Eriksson, and B. Johansson, Phys. Rev. B 51, 4813 (1995).

${ }^{33}$ M. Bienfait, P. Zeppenfeld, N. Dupont-Pavlovsky, M. Muris, M. R. Johnson, T. Wilson, M. DePies, and O. E. Vilches, Phys. Rev. B 70, 035410 (2004).

${ }^{34}$ W. Zhou, J. E. Fischer, P. A. Heiney, H. Fan, V. A. Davis, M. Pasquali, and R. E. Smalley, Phys. Rev. B 72, 045440 (2005). 